\title{
ABSTRACTS
}

\section{CONCEPT LEARNING AND INTELLIGENCE}

\section{— Effect of Task Complexity and Intuitiveness of the Relevant Dimension -}

\author{
Keiko Kuhara \\ University of Tokyo
}

by

$\&$

\author{
Giyoo Hatano \\ Dokkyo University
}

This study was aimed at investigating the relation of intelligence to concept learning. Two experiments were undertaken to clarify how different was the effct of task complexity and intuitiveness of the relevant dimension on learners' performance, depending on their intellectual development. In both experiments, Ss were to learn a simple concept which was defined by a value on a certain dimension. Ss were presented instances one at a time and required to guess whether it belonged to concept $\mathrm{A}$ or not. Confirming information was given immediately. Successive 10 correct responses were required as the criterion of learning. An instance could be described by values of 3 (form and number of figures, and width of a border) or 6 dimension (color of figure, color and position of a border in addition to 3 dimensions described above). Experimental variables were i) number of irrelevent dimensions $(2,5)$ and ii) intuitiveness of the relevant dimension (intuitively conspicuous--.-form of figures, intuitively less noticiable---width of a border).

Four groups of high (HI) and average intelli- gence (AI) 5-graders served as $\mathrm{Ss}$ in exp. I. In exp. II, average 6-graders and 4-graders were served as Ss, instead of $\mathrm{HI}$ and $\mathrm{AI}$.

The reseults were as follows:

1) The more was the number of irrelevant dimensions, the more difficult was learing. This inhibitive effect was marked regardless of the degree of intellectual development (MA).

2) If the relevant dimension was intuitively conspicuous, Ss learned the concept more easily. Contribution of this factor, however, was smaller among $\mathrm{HI}$ and 6-graders, i. e., intellectually more mature Ss.

3) It was hypothesized but was not statistically confirmed that intuitiveness of relevant dimension would greatly influenced on the performance of Ss when the number of irrelevant dimension was larger. The inhibitive effect of task complexity tended to be greater among 6-graders when the relevent dimension was conspicuous and smaller when it was less noticiable than among 4-graders. 